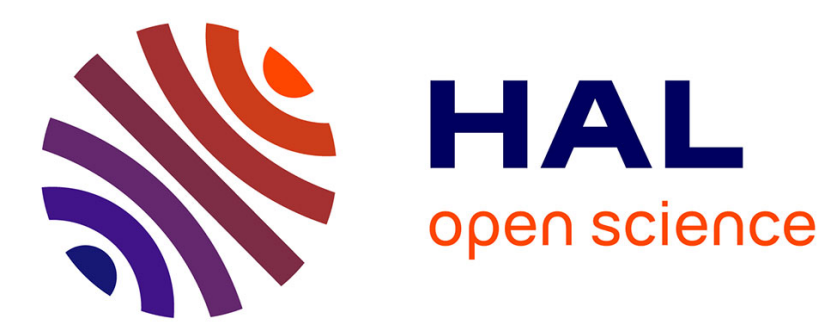

\title{
La rente et l'État rentier recouvrent-ils toute la réalité de l'Algérie d'aujourd'hui?
}

\author{
Fatiha Talahite
}

\section{To cite this version:}

Fatiha Talahite. La rente et l'État rentier recouvrent-ils toute la réalité de l'Algérie d'aujourd'hui?.

Revue Tiers Monde, 2012, 210, pp.143-160. hal-00717910

\section{HAL Id: hal-00717910 \\ https://hal.science/hal-00717910}

Submitted on 14 Jul 2012

HAL is a multi-disciplinary open access archive for the deposit and dissemination of scientific research documents, whether they are published or not. The documents may come from teaching and research institutions in France or abroad, or from public or private research centers.
L'archive ouverte pluridisciplinaire HAL, est destinée au dépôt et à la diffusion de documents scientifiques de niveau recherche, publiés ou non, émanant des établissements d'enseignement et de recherche français ou étrangers, des laboratoires publics ou privés. 


\title{
La rente et l'État rentier recouvrent-ils
}

\author{
toute la réalité de l'Algérie d'aujourd'hui ?'
}

\author{
Fatiha TALAHITE \\ Chercheure CNRS, \\ UMR 1742 CRESPPA \\ CNRS/Université Paris 8 \\ fatiha.talahite@gtm.cnrs.fr
}

\section{Résumé}

Partant du constat de l'hégémonie des concepts de rente et d'état rentier dans la littérature en sciences sociales et humaines sur l'Algérie, cet article revient sur le processus qui a mené à la domination des hydrocarbures sur l'économie et l'État algériens. Cette emprise s'est intensifiée depuis 1992, lorsque la réforme visant à légaliser le marché, par un processus de légitimation des institutions ayant conduit à une ouverture du champ politique, fut brutalement interrompue. Il montre comment un pouvoir illégitime, par son effet dissolvant du droit et de la légalité, pousse à l'informalisation de l'activité économique, pour ne laisser finalement apparaître comme seule réalité que la rente et l'état rentier. Il indique que le chemin pour en sortir et aller vers la réhabilitation de l'économie et des institutions, passe par un acte fondant dans les urnes une nouvelle légitimité.

Mots clé : Algérie, rente, État rentier, transition, marché intérieur.

\section{Are Rent and the Rentier State the Only Realities in Today's Algeria?}

\begin{abstract}
Using as a starting point the hegemonic place occupied by the concepts of rent and rentier state in the social sciences and humanities literature on Algeria, this article re-examines the process that led to the predominance of hydrocarbons on the Algerian economy and on the Algerian state. This hold by the hydrocarbons on the economy and the state has intensified since 1992 with the brutal interruption of the reform intended to legalise the market through a legitimation of the institutions, which led to an opening of the political field. It shows how an illegitimate power can lead to the informalizing of the economic activity as a result of its dissolving effect on law and legality, and eventually let appear the rent and the rentier state as the only realities. It indicates that the way to get out and move forward towards a rehabilitation of the economy and the institutions is through the ballot box and the forging of a new legitimacy.
\end{abstract}

Key words: Algeria, rent, rentier state, transition, domestic market.

\footnotetext{
${ }^{1}$ Une version de cet article a été publiée dans la Revue Tiers Monde, n²10, avril-juin 2012.
} 
En Algérie, la rente des hydrocarbures, qui représente plus de la moitié du revenu national et la quasi-totalité des exportations, tend à faire apparaître l'ensemble de la nation comme une «classe» de rentiers de l'économie internationale ${ }^{2}$. Comment cette rente a-t-elle pu prendre tant d'importance au point de faire passer au second plan les autres formes de production ${ }^{3}$, de parasiter tous les rouages de la vie économique, sociale et politique ? Si cette notion a recouvert tous les aspects de la société, de l'économie et de l'État, ne laissant pas la place à d'autres conceptualisations, est-ce imputable à la prégnance d'un concept holiste qui englobe tout, ou n'existe-t-il effectivement plus aucune réalité dans l'Algérie d'aujourd'hui qui ne serait soluble dans la rente?

\section{La centralité des concepts de rente et d'état rentier}

La littérature récente en sciences humaines et sociales sur l'Algérie consacre la centralité du concept de rente. Au niveau de l'analyse de l'État notamment, la théorie de l'état rentier a supplanté toutes les autres ${ }^{4}$.

\section{L'État rentier}

Considérée comme «l'une des contributions majeurs des études de la région du MoyenOrient à la science politique » (Anderson, 1987), cette notion, élaborée à partir des cas de l'état iranien (Mahdavi, 1970) puis arabe (Beblawi, Luciani ; 1987, Beblawi, 1990), a connu un succès foudroyant au point que rares sont aujourd'hui les écrits en sciences sociales et humaines sur la région où on ne la retrouve pas. Si elle a pu produire des effets de savoir (Catusse, 2006), la rançon de son succès tend à en faire une notion passe-partout au contenu de plus en plus imprécis. En économie, elle est utilisée aussi bien par les néo-classiques et néo-institutionnalistes que les courants hétérodoxes (institutionnalistes, structuralistes, régulationnistes), qui s'appuient pourtant sur des théories différentes de la rente (rent seeking d'un côté, rente ricardienne ou marxienne de l'autre).

Nous avons déjà proposé une généalogie de cette notion qui réactualise en lui donnant un fondement économique moderne, la vieille vision occidentale du despotisme oriental, allant

\footnotetext{
${ }^{2}$ Cependant, la rente nette par habitant diminue. En termes réels, elle ne représente en 2000 que $40 \%$ du niveau atteint en 1981.

${ }^{3}$ L'informel dans l'économie, dont l'évaluation diffère selon la définition et la technique adoptée, atteindrait plus de $40 \%$ du PIB. Pour une estimation de la production non prise en compte par la comptabilité nationale à partir de la consommation d'électricité voir Bouklia-Hassane, Talahite (2008). Pour un point récent sur la question, voir Bellache (2010).

${ }^{4}$ Dans la définition de Beblawi (1990), tous les pays arabes sont rentiers, les non-pétroliers étant définis comme semi-rentiers.
} 
de Montesquieu à Witfogel, en passant par Marx et Weber (Talahite, 2006). Elle véhicule des clichés orientalistes intériorisés par les élites technocratiques du MENA, sous la caution d'un discours d'économie politique neutre et scientifique. Appliquée à l'Algérie, cette vision tend à plaquer l'image d'un État autoritaire héritier du despotisme oriental, ignorant l'histoire singulière de cet État directement issu de l'administration coloniale ${ }^{5}$, auquel se sont greffés des éléments du modèle soviétique, dont il faudrait cependant évaluer ce qu'il reste depuis leur démantèlement dans les années 1990. Quant aux institutions de la régence turque, dévastées lors de la conquête ${ }^{6}$, il n'en reste plus grand-chose, si ce n'est quelques lambeaux dans la mémoire collective. Aussi, lorsque Auty et Gelb (2001) opposent l'État algérien «fortement centralisé » à celui du Nigeria où la compétition pour les rentes a mené à la guerre civile $^{7}$, cela relève plus d'un cliché que d'une observation sur le terrain ${ }^{8}$.

Dans la théorie de l'état rentier, la rente est assimilée à un revenu extérieur. Pour le définir, Beblawi utilise le terme anglais d'external rent ${ }^{9}$. Il y inclut l'aide politique et militaire, les royalties du Canal de Suez et des oléoducs pour les pays de passage et même les transferts de salaires des travailleurs émigrés (Beblawi, 1990 : 97). Concernant ces derniers, d'une part, il s'agit de revenus du travail, d'autre part, ils ne vont pas à l'État. En fait, l'importance accordée à ces transferts est liée au fait qu'ils donnent lieu à une conversion de devises en monnaie locale et qu'ils jouent ainsi un rôle dans l'ajustement des balances des paiements ${ }^{10}$.

Si c'est en tant que revenu extérieur que la rente des hydrocarbures est au fondement de l'État rentier, cela conduit à se demander jusqu'à quel point cette construction théorique s'appuie sur une théorie de la rente. Il apparaît qu'elle s'appuie sur au moins deux théories de la rente : celle issue de l'économie politique classique, d'une part, celle du rent seeking issue du Public Choice d'autre part. Une troisième théorie, le modèle du Dutch Disease, analyse les effets de

\footnotetext{
${ }^{5}$ Acemoglu et al. (2001) prennent en compte la colonisation dans la détermination des institutions et de leurs effets sur le développement - notamment à l'aune de la protection de la propriété - et la persistance de cette détermination après la décolonisation. Le cas de l'Algérie est atypique : l'implantation d'une forte colonie européenne fait qu'il ne peut être considéré comme un extractive state, mais le départ massif des pieds-noirs à l'indépendance ne le range pas non plus dans la «néo-Europe».

${ }^{6}$ Les archives de cet État ont été totalement détruites lors de l'occupation d'Alger en 1830 (Weber, 2010).

7 "Resource rents can, for example, help to consolidate tendencies toward a strongly centralized state (as in Algeria); or alternatively, competition over rents can spark civil war (as in Nigeria) and the disintegration of the state" (Auty, Gelb, 2001:126). Pour Woolcock et al. (2001:82): "Two variations can be identified. In the first, the state owns the rentier capitalists so that a regime of rentier autocrats emerges, as with Algeria and Nigeria; in the second, the rentier capitalists effectively own the state, as in Angola and El Salvador, and oligarchic regimes emerge".

${ }^{8}$ Apparemment, ces auteurs n'ont pas entendu parler de la guerre civile en Algérie!

${ }^{9}$ Rappellons que rent en anglais signifie revenu, ce qui introduit un biais dans la traduction en Français de rentier state.

${ }^{10}$ Beblawi y évoque l'Algérie, non comme pays pétrolier, mais parmi ceux où ces remittances sont importantes. Pourtant, ce n'était déjà plus le cas : si en 1976, près de la moitié des transferts financiers privés de France vers le Maghreb provenaient des algériens, en 1986 cette proportion a chuté à 0,4 \% (Collyer, 2012).
} 
la rente des hydrocarbures sur l'économie, en tant qu'elle est considérée comme un revenu extérieur dépendant de facteurs exogènes à la nation.

\section{La rente des hydrocarbures}

Le concept de rente, qui nous vient de la théorie de la rente agricole dans l'économie politique classique, est à la fois économique et politique. Chez Ricardo, le surproduit est partagé entre salaires, profits et rentes, chaque revenu étant associé à une classe économique donnée. Marx fera de la rente un rapport social, à côté du capital et du salariat, fondant économiquement une classe sociale, les rentiers de la terre.

C'est le marché qui fait apparaître la rente, comme distincte des salaires et profits ${ }^{11}$. La pertinence de cette notion est liée à l'existence d'un marché sur lequel celle-ci est générée. Même la théorie du rent seeking (Krueger, 1974 ; Tullock, Mason, 2005) issue du courant du Public Choice, qui s'intéresse aux comportements microéconomique de recherche de rentes induits par des distorsions de marché (dues notamment à l'intervention étatique) et aux pertes que ces rentes entraînent pour l'économie ou la société (Bhagwati, 1982, 1996), prend le marché comme référence à partir de laquelle identifier l'existence de rentes. Tant que celui-ci n'existe pas, on ne peut parler de rente ${ }^{12}$.

Chez les classiques, la rente agricole est issue d'une valorisation sur le marché domestique. Mais la rente des hydrocarbures, due à l'écart entre leur coût d'extraction et leur valeur de marché, ne provient pas du marché national. Comme revenu extérieur, elle est prélevée sur la valeur produite à l'échelle de l'économie mondiale, sur un marché globalisé. C'est en tant qu'ils sont valorisés sur le marché extérieur que les hydrocarbures procurent une rente. Ainsi, l'allocation de la valeur sur le marché mondialisé génère une rente pour le propriétaire ${ }^{13}$ de la ressource (l'État algérien). Cette rente est captée par la fiscalité pétrolière. Une partie revient à l'entreprise nationale Sonatrach en tant qu'elle jouit une position de monopole dans l'exploitation de ces ressources. On peut d'ailleurs y voir une rente de monopole, distincte

\footnotetext{
${ }^{11}$ «La rente au sens de Ricardo n'est possible que dans le cadre de la concurrence capitaliste (..). Au lieu d'attacher l'homme à la nature, [elle] n'a fait que rattacher l'exploitation de la terre à la concurrence. Une fois constituée en rente, la propriété foncière elle même est le résultat de la concurrence, puisque dès lors elle dépend de la valeur vénale des produits agricoles » (Marx, 1965 :123). Comme Ricardo, Marx considère la rente comme «illégitime » dans le sens où elle ne rémunère pas une participation à la production, mais il étend cette illégitimité aux droits de propriété privée, qui masquent «le fait initial de la conquête sous le manteau du 'droit naturel'» (ibid. : 1476).

${ }^{12} \mathrm{~A}$ propos des notions d'impôt, redevance, tribut ou rente foncière, qu'il définissait comme des «prélèvements payés par le paysan ou le cultivateur, le producteur de denrée alimentaire, à une autorité reconnue », Hicks (1971 : 31) écrivait qu'« en l'absence d'un marché, on ne distingue pas ces termes l'un de l'autre ».

${ }^{13}$ La connaissance des rentes est liée à l'existence de droits de propriété clairement établis et à l'enregistrement des transactions. Sinon, il est difficile d'établir l'existence et le montant des rentes, comme c'est le cas dans l'économie informelle.
} 
dans sa définition de la rente dite naturelle, liée à la rareté. L'enjeu de la loi nº 05-07 sur les hydrocarbures promulguée en 2005 était précisément de supprimer cette rente en mettant la Sonatrach en concurrence avec d'autres compagnies, algériennes et étrangères, pour la recherche et la production des hydrocarbures (Aïnas et al., 2012) ${ }^{14}$.

La redistribution de ce revenu par l'État, dans la mesure où elle passe par la dépense publique, se fait sous forme de transferts. Dans quel cas cette redistribution est-elle génératrice de rentes? Lorsque les transferts (allocations, subventions) se font selon des règles claires et démocratiquement établies, on parle d'État providence. Mais s'il est difficile d'évoquer l'État providence dans un système non démocratique, peut-on pour autant appeler rentes tous les revenus distribués par un État non démocratique? Quid alors des salaires des fonctionnaires, des transferts sociaux?

Si la rente énergétique est à l'origine du revenu de l'État, donc indirectement des rentes issues de sa redistribution par l'État, celles-ci ne sont pas de même nature. Alors que c'est le marché (mondialisé) qui génère la première, analysée comme une « rente naturelle », sa redistribution par l'État se fait sous forme de transferts. Les modalités de cette redistribution (prix administrés, subventions, allocations..) peuvent être génératrices de rentes non naturelles et provoquer dans l'économie des distorsions de marché favorables au rent seeking.

\section{Le rent seeking}

La théorie du rent seeking montre comment les rentes résultant de l'intervention de l'État et des institutions, notamment du fait de la législation, ont pour effets une réduction de la production. Pour Bhagwati (1982), ce sont des activités de recherche de profits directement improductive (DUP) provoquant une perte directe pour le revenu national, et, indirectement, une hausse des taux de profit dans les secteurs rentiers, où sont attirés les capitaux, au détriment d'autres secteurs où les profits espérés sont plus faibles, ce qui a également pour résultat une diminution de la production totale. Ainsi, le rent seeking n'est pas un jeu à somme nulle pour l'économie. La théorie du Public Choice distingue d'ailleurs, à l'opposé du rent-seeking, le profit seeking, activité dont le gain social marginal est supérieur au gain privé marginal (Buchanan, 1980).

Ce n'est pas la rente pétrolière qui crée ces effets, mais la manière dont l'État et les institutions interviennent dans l'économie. La rente énergétique permet dans une certaine

\footnotetext{
${ }^{14}$ Cette concurrence devait être régulée par deux agences - l'Autorité de régulation des hydrocarbures (ARH) et L'Agence nationale pour la valorisation des ressources en hydrocarbures (ALNAFT) - dont les missions sont devenues en partie caduques avec l'élimination de cette concurrence par l'amendement de la loi par ordonnance présidentielle en 2006.
} 
mesure de compenser la baisse de production induite par le rent seeking, et elle fournit également à l'État le moyen de financer les déficits éventuels causés par son intervention dans l'économie. Mais le rent seeking peut se développer en l'absence de rente pétrolière. Ainsi, en Algérie, lorsque les ressources pétrolières ont diminué de manière drastique, dans les années 1990, l'activité de rent seeking n'a pas diminué pour autant, au contraire, c'est à cette période qu'elle s'est généralisée (Dillman, 2000). De même avec le gel d'une partie des revenus des hydrocarbures dans le Fonds de stabilisation des réserves depuis 2001 (Talahite, Hammadache, 2011).

La théorie du rent seeking a été utilisée pour donner un fondement micro-économique à l'approche structuraliste et institutionnaliste du développement (Khan, Jomo, 2000).

\section{Le Dutch Disease}

Considéré sous son aspect de revenu extérieur, dont la détermination et les variations dépendant de facteurs exogènes à la nation, les effets de la rente des hydrocarbures sur l'économie sont souvent analysés à l'aide du modèle du Dutch disease, qui permet d'étudier les effets d'un windfall (manne), et des chocs qu'il produit sur l'économie. Conçue à l'origine pour expliquer la relation entre inflation et taux de change dans de «petites » économies ouvertes développées sans contrôle étatique (Australie, Pays-Bas, Irlande) soumises aux effets d'une manne permanente, cette théorie n'était pas transposable telle quelle aux économies en développement, à cause de différences structurelles (absence de marchés financiers, ouverture de l'économie, chocs commerciaux externes temporaires, importance du contrôle de l'État sur l'économie). Bevan et al. (1990) en ont élaborée une version adaptée aux petites économies ouvertes des pays en développement soumises à un contrôle étatique. Leur modèle vise à dépasser à la fois la faible attention apportée aux institutions par la théorie néo-classique, et l'absence de fondements micro-économiques des théories structuralistes. Appliquée à l'étude des effets au Kenya et en Tanzanie (1976-78) du « coffee boom » provoqué par le gel de la récolte de café au Brésil en 1975, ce modèle aboutit à distinguer deux régimes de contrôle étatique: l'un dit « compatible » débouche sur un équilibre soutenable bien que sous-optimal (Kenya), tandis que l'autre (Tanzanie), « incompatible », provoque une contraction et une «implosion» de l'économie. Les effets d'un démantèlement du contrôle des prix diffèreront selon le régime : souhaitable dans le régime compatible, il est considéré comme risqué dans le second où il est préconisé de procéder par étapes, avec des réformes de structure préalables. Selon cette typologie, l'Algérie jusqu'à la fin des années 1980 relevait du modèle 
incompatible, ce qui expliquerait les dégâts causés par la politique de libéralisation des prix menée au début des années 1990, sous la pression du FMI.

L'application du modèle du Dutch Disease a été récemment étendue à l'histoire économique du développement. Williamson (2011) montre à partir des cas de l'Inde, du Moyen-Orient sous les Ottomans et du Mexique, comment des processus d'industrialisation amorcés au 19e siècle ont été interrompus par la hausse des prix des matières premières provoquée par le boom de la demande des premiers pays industrialisés (Europe de l'Ouest et États-Unis), qui a entraîné non seulement une spécialisation internationale (selon la théorie ricardienne) mais également une désindustrialisation. C'est ce phénomène qui est, selon lui, à l'origine de la grande divergence entre le centre et la périphérie. Il faut cependant préciser qu'à cette époque, si ces pays connaissent effectivement un début d'industrialisation, ce sont les pays occidentaux, et en premier lieu l'Angleterre, qui contrôlent les échanges mondiaux de matières premières, dans le contexte de la première mondialisation. Cette analyse historique peut éclairer les effets du boom actuel sur les matières premières provoqué par l'accélération de la croissance industrielle dans les pays émergents, en termes de spécialisation internationale et de désindustrialisation dans les autres pays en développement.

\section{Rente extérieure et émergence du marché intérieur}

\section{L'articulation entre extérieur et intérieur, politique et économique}

Une difficulté réside dans la juxtaposition de deux espaces hétérogènes - d'un côté, le marché mondialisé où la rente est générée, de l'autre, sa redistribution à l'intérieur du pays - et dans la transposition, dans le second espace, d'un concept qui trouve sa pertinence dans le premier. Deux espaces que la catégorie de rente n'articule pas entre eux parce que la rente des hydrocarbures est radicalement différente des rentes générées par sa redistribution interne.

Quant à l'articulation entre l'économique et le politique, elle est asymétrique. La théorie de l'état rentier, en effet, qui nous vient de la science politique, s'intéresse en priorité aux aspects politiques de la répartition du revenu des hydrocarbures à l'intérieur de la nation, à travers les luttes de pouvoirs et les enjeux clientélistes. Par contre, elle tend à ne considérer la génération de ce revenu que sous son aspect de rente naturelle, en écartant sa dimension politique, les rapports de pouvoir à l'échelle internationale en tant qu'ils ont un effet sur le partage de cette rente. En isolant ainsi les rapports de pouvoir à l'intérieur de la nation des interférences externes, les connexions entre modalités internes et externes de répartition de la rente (notamment à propos de la corruption) sont masquées. Or la redistribution interne à la nation 
du revenu des hydrocarbures n'est que le bout de la chaîne, le dernier maillon d'un procès dont la dynamique est mondialisée ${ }^{15}$. A travers la rente des hydrocarbures, l'ensemble de la nation est relié au marché mondial. Au fur et à mesure que, sous la pression de la demande externe (mais également d'autres facteurs qu'il s'agit d'identifier) cette rente prend de l'importance dans le revenu du pays, elle fait passer au second plan les autres formes de production et tout le pays est emporté dans le tourbillon de la valorisation externe des hydrocarbures.

La théorie du rentiérisme, lorsqu'elle va jusqu'à considérer la totalité du revenu redistribué par l'État comme une rente pour ceux qui en bénéficient, assimilée à la rente des hydrocarbures, est réductrice : d'abord parce qu'elle tend à confondre cause (rente générée sur un marché mondialisé) et effets (rentes générées sur le marché domestique) ; ensuite parce qu'elle fait l'impasse sur la différence de nature entre ces rentes ; enfin parce qu'elle conduit à assimiler l'état propriétaire de la rente à la nation toute entière et en son sein à chaque individu bénéficiaire de la redistribution étatique. Il est difficile de l'utiliser pour l'analyse des rapports de causalité internes à la nation, tant elle donne de cette dernière une représentation inconsistante.

\section{Rente urbaine et émergence du marché immobilier foncier et locatif}

Pour étudier l'émergence du marché intérieur, il importe de distinguer entre rente pétrolière et les autres rentes potentiellement générées sur le marché domestique, comme la rente foncière ou locative urbaine. Pour Safar Zitoun (2012), en Algérie «les rentes urbaines [sont des] formes dérivées de la rente pétrolière ». Or les rentes liées à l'allocation administrée des logements et leur vente à des prix subventionnés, conséquence d'une gestion étatique déficitaire du parc immobilier, sont distinctes à la fois de la rente des hydrocarbures et des rentes urbaines (locative ou foncière). Car, comme l'écrit à juste titre cet auteur, les « rentes de localisation incorporées dans les biens immobiliers (..) ne s'expriment qu'à la faveur de leur mise en circulation sur le marché ». A moins que l'on analyse tous les marchés comme dérivés de la rente pétrolière, en considérant que sur ces marchés, tous les biens et tous les revenus proviendraient de la rente pétrolière. Mais dans ce cas, tout est dans tout, la rente est un concept totalisant.

Par l'opération de liquidation des biens de l'État en 1980, l'État visait précisément à se désengager de cette gestion. L'accès à la propriété, à partir du moment où il débouche sur 
l'émergence d'un marché, va dans le sens de supprimer les rentes liée à l'accès privilégié et discrétionnaire aux ressources de l'État, ne laissant apparaître que la rente urbaine due à la localisation. Dès lors, il devient possible de mettre en place une politique fiscale consistant à prélever sur cette rente pour financer une politique de logement social. Safar Zitoun (2012) montre comment ce marché peine à émerger, du fait de la difficulté de l'État à se désengager. Il en voit la cause principale dans « le pacte implicite noué autour de la distribution du butin de guerre colonial [qui] continue de distiller ses effets structurels sur l'ensemble des pratiques des acteurs $»^{16}$.

\section{Historiciser l'État rentier}

Cet auteur fait de l'appropriation d'un «butin de guerre » scellant un «pacte patrimonial originel », la base sur laquelle s'est édifié le nouvel État algérien à l'indépendance ${ }^{17}$, avec l'appropriation des logements et des terres laissés par les colons. Ce moment inaugural est probablement décisif, mais il faudrait, pour lui donner toute sa densité historique, le rapporter à un autre fait historique auquel il fait écho en négatif, la confiscation des logements, des biens et des terres des algériens lors de la conquête française, notamment lors de l'installation de l'armée dans la ville d'Alger, puis les expropriations massives durant la première phase de la colonisation. Weber (2010) montre comment s'est constitué le patrimoine foncier colonial, et tout d'abord le domaine foncier de l'État, par l'arbitraire et la violence, mais aussi par la $10 i^{18}$. Ces mesures d'expropriation, qui provoquèrent un effondrement du marché immobilier, furent rendues possibles par le fait que l'ordre juridique concernant le domaine foncier répondait sur le territoire algérien à des normes radicalement différentes de celles qui régissaient le droit français. «La propriété foncière, qu'elle soit publique ou privée, reposait, dans ses grandes orientations, sur le droit coutumier et le droit islamique » (ibid. 93). On comprend mieux pourquoi à l'indépendance, sous couvert de nationalisation, s'est installée une situation de non-droit. Car s'il s'agissait d'abolir l'ordre colonial, l'ancien ordre n'était pas restauré pour autant. Cette question, enfouie pendant toute la période socialiste, va ressurgir lors du lancement des réformes.

\footnotetext{
${ }^{16}$ Il y aurait d'autres explications, dont la croissance démographique et l'explosion de la demande, qui fait que l'offre de logements est insuffisante et que l'État doit contribuer à combler le déficit. Il y a aussi une demande spéculative, en l'absence de marchés financiers et d'opportunités d'investissement. Enfin, il reste la vieille question, déjà traitée en 1887 par Engels, de l'impossibilité d'un accès généralisé à la propriété de leur logement par les classes populaires, qui explique que l'État doive construire des logements sociaux.

${ }^{17} \mathrm{La}$ nationalisation des biens coloniaux à l'indépendance, visant à les soustraire à l'occupation et l'appropriation sauvages, n'aboutit pas à la création d'un véritable secteur public. Il aurait fallu que « les procédures de décision [soient] publiques, nationales, et représentatives » (Henni, 1999).

${ }^{18}$ Par des procédés similaires à ceux utilisés en Amérique pour déposséder les amérindiens (Roberston, 2005).
} 
Les institutions, dont les droits de propriété, sont avant tout un héritage, elles nous viennent du passé. Pour y toucher par des réformes, il faut une légitimité. C'est pourquoi les injonctions des experts internationaux à améliorer les institutions et renforcer les droits de propriété apparaissent comme naïves ou velléitaires. La théorie économique prend acte à sa manière de cet héritage, lorsqu'elle parle de dotations initiales sur un marché, c'est-à-dire de la manière dont sont répartis les facteurs de production. De son côté, l'histoire du capitalisme nous enseigne comment ces « dotations » sont le résultat d'une accumulation primitive faite de violence et de spoliation. En Algérie, cette histoire - l'entrée dans la modernité capitaliste - commence avec la colonisation. Après l'indépendance, avec la confiscation puis la redistribution par l'État des logements, de la terre et d'autres ressources, puis des revenus des hydrocarbures, s'est opérée une «accumulation primitive », sous le couvert de la légitimité révolutionnaire au fondement $\mathrm{du} \ll$ pacte patrimonial ${ }^{19}$.

\section{3. la transformation post-socialiste}

L'emprise des hydrocarbures sur l'économie puis sur l'ensemble du pays, État et société inclus, ne s'est pas faite du jour au lendemain. Il importe de revenir sur les différentes étapes de ce processus, qui n'est pas linéaire. A certains moments, comme lors de l'industrialisation dans les années 1970 ou de la réforme de 1988-1991, la nation a su se mobiliser, sous des modalités différentes, pour reprendre la maîtrise de ses ressources et de son destin. La configuration des coalitions et élites au pouvoir a alors joué un rôle. Par contre, cette emprise s'est renforcée dans les temps de vulnérabilité, durant lesquelles les choses ont basculé. Après la baisse des prix du pétrole en 1985, les dirigeants n'ont pas su anticiper ni maîtriser la dégradation de l'économie, mais ils ne disposaient pas non plus des leviers de politique économique ni des institutions pour faire face. Cette période a débouché sur les réformes de 1988.

\section{La réforme de 1988-1991 : une période charnière}

De l'indépendance jusqu'aux années 1987-1988, l'économie de l'Algérie, orientée vers un projet de développement autocentré, était caractérisée par une prégnance de l'État et de l'administration sur l'économie, une répression des mécanismes du marché ainsi qu'une

${ }^{19}$ La terre est considérée comme la « compensation légitime pour les combattants de la libération ou leur famille, représente symboliquement le prix du sang versé pour l'ensemble de la nation, justifiant son appropriation par des cercles du pouvoir, ce qui contredit un autre principe souvent réaffirmé, celui de la propriété de l'usufruit à ceux qui mettent la terre en valeur » (Omari et al., 2012). 
velléité de rupture avec l'économie mondiale capitaliste. La période qui suit est celle d'une ouverture à la fois interne, sur le marché et ses institutions, et internationale, à travers la recherche d'une intégration à l'économie régionale et mondiale.

Les années 1988-1991, à la charnière entre ces deux périodes, sont le moment d'une expérience originale de réforme dont on a encore peu analysé tous les aspects. Bien que prématurément interrompue en juin 1991 avec le départ du gouvernement réformateur et surtout en janvier 1992, avec le coup d'État, l'annulation des élections législatives et la promulgation de l'état d'urgence, cette réforme, par la dynamique qu'elle a enclenchée et les questions qu'elle a soulevées, a tracé le cadre, globalement non dépassé à ce jour, dans lequel penser le processus de transformation économique en Algérie.

Portée par un courant minoritaire au sein du FLN appelé au gouvernement à la faveur d'une situation économique devenue critique, la réforme, d'abord pensée comme économique, avait pour premier objectif une régulation plus efficace, s'appuyant sur des mécanismes de marché. De fil en aiguille, elle a débouché sur un projet plus vaste, englobant la réforme des institutions. Il s'agissait d'harmoniser les règles de droit entre les secteurs privé et étatique de l'économie et introduire de la concurrence entre eux ainsi qu'au sein du secteur des entreprises publiques, sans toutefois remettre en cause la propriété étatique. A ce stade, il n'était question ni de privatisations ni de libéralisation du commerce extérieur. Cette réforme s'inspirait du projet de socialisme de marché alors en débat dans les pays de l'Est avant la chute du mur de Berlin ${ }^{20}$, notamment en Hongrie (Kornaï, 1990), ainsi que du modèle français de gestion des entreprises nationalisées dans un environnement de marché. Les réformes monétaire et des finances publiques étaient conçues « comme une tentative de réhabilitation préalable des fonctions régulatrices de l'État en vue du gouvernement d'un marché à venir et d'une ouverture internationale » (Henni, 2009 : 37). Dans la mesure où il s'agissait de mettre en place les règles et institutions du marché, la réforme avait un contenu juridique primordial $^{21}$. Dès lors la question de la légitimité de l'instance législative se posait, et donc de la nécessité d'une réforme institutionnelle.

\footnotetext{
${ }^{20}$ C'était alors la Pereströ̈ca en URSS (de 1985 jusqu'à fin 1991).

${ }^{21}$ Les réformateurs, tout comme les dissidents au régime communiste, étaient sensibles à «la nécessité d'un régime de Droit au sens de la déclaration universelle de 1948, c'est-à-dire d'une instance Tierce, incarnée depuis les Temps modernes par la figure de l'État, qui transcende les intérêts particuliers et est garante des droits des individus » (Supiot, 2010: 40). Cette vision fut balayée après la chute du mur par la conversion des communistes au Marché et leur ralliement à l'ultralibéralisme, « qui partage avec le socialisme scientifique la même foi dans la validité universelle des 'lois de l'économie' et la même détermination à soustraire ces lois au champ de la délibération politique » (Supiot, $2010: 41$ ).
} 
Cependant, autant le volet économique avait pu être pensé ${ }^{22}$, autant les mesures politiques ont été prises dans la précipitation, sous la pression des évènements internes et externes ${ }^{23}$. Les réformateurs avaient envisagé une ouverture et une démocratisation du $\operatorname{FLN}^{24}$ (par l'institution de courants en son sein, sur le modèle du parti socialiste français). Dans leur vision, à une transformation progressive de l'économie planifiée et administrée en économie de marché, devait correspondre un passage progressif de la légitimité révolutionnaire incarnée par le FLN, à une légitimité fondée sur de nouvelles institutions, que le parti historique aurait été en charge d'initier, garantissant ainsi une continuité institutionnelle, dans une transition qui aurait pu évoluer vers un modèle à la chinoise ou à la vietnamienne. Ce faisant, ils auraient pris la tête d'un ambitieux projet, dépassant largement les prérogatives qui leur étaient concédées. Aussi, au moment où les choses se débloquent au sein du FLN et que le courant réformateur monte en puissance, les autorités décident de rebattre les cartes en faisant adopter par l'assemblée nationale (issue du FLN) une révision de la constitution reconnaissant le pluripartisme, supprimant de fait le monopole du FLN. Le gouvernement réformateur a pris en main cette réforme, s'adaptant à la nouvelle donne créée par la révolte d'octobre 1988. A la lumière de ce qui s'est passé par la suite, on peut supposer que l'enjeu était bien de lui ôter le pouvoir et la légitimité que lui aurait conférée un FLN rénové, en le réduisant à une équipe de technocrates en charge des réformes économiques.

On a là une configuration particulière du constat que fait Rivlin (2009 : 38) : «Si l'absence de réforme politique peut s'expliquer de manière simple par le désir de ceux qui sont au pouvoir de le garder, l'échec à introduire des réformes économiques est plus compliqué. Car la faillite économique peut aussi menacer le pouvoir et faire tomber le régime. La réforme peut être le moyen d'éviter cela ${ }^{25}$. Si les tenants du pouvoir, après s'y être longtemps opposés, ont fini par se résoudre à réformer l'économie, ils n'étaient pas prêts à accepter que les réformateurs bénéficient d'une double légitimité : celle historique du FLN et celle que leur aurait conféré la réforme. Le problème est que, privée de son instrument politique - le parti rénové- la réforme ne pouvait être menée à bien. De fait, dès premières élections pluralistes (élections locales de

\footnotetext{
${ }^{22}$ Voir les Cahiers de la réforme (Hadj Nacer, 1989, 1990).

${ }^{23}$ A la $16^{\mathrm{e}}$ conférence des chefs d'État de France et d'Afrique (La Baule, 20 juin 1990), le président Mitterrand prononça un discours contre le régime de parti unique, faisant de la démocratisation une condition de l'aide au développement. Pour certains, cela a pu infléchir la trajectoire des réformes en Algérie et accélérer le cours des évènements, en mettant une forte pression externe sur la réforme politique.

${ }^{24}$ Front de Libération Nationale.

25 «If the absence of political reforms can be simplistically explained as a desire by those in authority not to relinquish power, the failure to introduce more economic reforms is more puzzling. Economic failure can also threaten the power structure and bring down the regime; reform can therefore be a way of avoiding that ».
} 
1990) l'entrée en scène de nouveaux acteurs, dont le FIS $^{26}$, a totalement bouleversé le paysage politique, créant une situation d'instabilité et d'incertitude défavorable à la réforme économique, désormais reléguée au second plan. D'autant que, parallèlement, les difficultés de balance des paiements ${ }^{27}$ faisaient peser la menace d'un rééchelonnement de la dette extérieure conditionné à un programme d'ajustement structurel qui aurait lié les mains du gouvernement, le privant de son autonomie pour mener la réforme. Le nouveau gouverneur de la Banque d'Algérie (Hadj Nacer, 2010), convaincu que la nature à court terme de la dette et la solvabilité du pays à long terme - du fait de ses ressources énergétiques - permettaient d'éviter le rééchelonnement, tenta vainement d'obtenir un «reprofilage» de la dette (négociations bilatérale avec les créanciers) ${ }^{28}$, tout en négociant un accord stand by avec le FMI (juin 1991). En juillet, le gouvernement était contraint de démissionner ${ }^{29}$. Malgré les multiples tentatives des autorités pour reprendre le contrôle de la situation - notamment en manipulant les élections législatives par le biais du découpage électoral - l'armée interrompit le processus dès l'annonce des résultats du premier tour.

Cette expérience illustre la complexité des relations entre réforme économique et politique, ainsi qu'entre facteurs internes et externes. Les réformateurs voulaient se donner les instruments de politique économique qui leur auraient permis de maîtriser l'économie et d'éviter la dérive de l'endettement. Pour cela, ils furent amenés à mettre en place les institutions du marché. Ce faisant, ils ouvraient la boîte de Pandore, non seulement de la réforme politique, qui s'est retournée contre eux, mais aussi de l'ouverture extérieure. Mais surtout, ce moment a fait surgir une dimension explosive de la réforme. L'émergence de l'Islam dans l'espace de la compétition politique et économique extirpait une question enfouie depuis la conquête de l'Algérie et évitée à l'indépendance, au moment de la mise en place de l'état algérien : le caractère incontournable de la référence normative à l'Islam lorsqu'il s'agit des fondements des institutions. Cette question, qui est celle des montages dogmatiques (Legendre, 1999), se situe en amont de celle de la laïcité ou de la sécularisation, qui porte sur la manière d'organiser la relation au religieux au sein d'institutions déjà là. Saura-t-on aujourd'hui en reconnaître l'enjeu, à travers les processus enclenchés par les printemps arabes, et entendre les enseignements tragiques de l'expérience algérienne ?

\footnotetext{
${ }^{26}$ Front Islamique du Salut.

${ }^{27}$ En 1988, l'Algérie avait sollicité du FMI une aide à la balance des paiements.

${ }^{28}$ L'échec de cette démarche fut dû au refus de la France, principal créancier au sein du Club de Paris.

${ }^{29}$ Certaines mesures, comme la réforme de l'impôt (Henni, 2009), menaçaient les intérêts de la base sociale du régime.
} 


\section{Depuis 1992 : la transition désinstituée}

A partir de 1992, l’Algérie entre dans une «transition désinstituée » (Sgard, 1997). Le chantier ouvert par la réforme est abandonné, mais l'ancien système n'est pas restauré pour autant, malgré la tentative du gouvernement de Abdessalam (juillet 1992- juillet 1993) et son « économie de guerre ». L'ouverture au marché se poursuit sans le volet institutionnel, ce qui débouche sur une libéralisation sauvage, avec, dans le même temps, émergence de monopoles privés qui s'accaparent notamment les réseaux d'importation. La guerre civile ${ }^{30}$, qui s'achève avec la loi du 13 juillet 1999 sur la Concorde civile est, après le Rwanda, l'un des conflits les plus meurtriers de l'après guerre froide (on parle de 150 à 200 mille morts et disparus ${ }^{31}$ ). A nos jours, aucune commission d'enquête indépendante, aucun tribunal digne de ce nom n'a fait la lumière sur les grands massacres, les milliers de disparus, les violations systématiques des droits humains. La guerre contre le terrorisme a recouvert d'un voile noir ce qui a commencé par l'interruption par l'armée d'un processus démocratique et le refus de reconnaître la légitimité sortie des urnes. La Concorde civile, si elle a permis l'atténuation de la violence, n'a été, sur le plan de la justice, qu'un simulacre, consacrant l'impunité de ceux qui, dans la chaîne de commandement, portent la responsabilité des violations des droits de l'homme et des crimes contre l'humanité perpétrés à large échelle sous couvert de lutte contre le terrorisme $\mathrm{e}^{32}$. Le dispositif mis en place, qui organise la relation entre façade institutionnelle et services de sécurité, vise d'abord à garantir cette impunité, notamment depuis les avancées de la justice internationale en matière de violation des droits humains. Il consacre l'extension du non-droit à tous les niveaux de l'État et de la société. Toute la politique menée depuis peut s'analyser au prisme de cette exigence fondamentale : la politique étrangère, édifiée sur ce que Keenan (2012) appelle la «rente du terrorisme», mais également la politique mercantiliste d'accumulation de réserves de change, sacrifiant le développement à la sécurité financière extérieure, afin d'éviter de prêter le flanc aux pressions externes en cas de difficultés de balance des paiements.

C'est à ce moment que l'Algérie est emportée dans une spirale de dépendance extrême aux hydrocarbures. Après le coup d'État de 1992 et l'interruption des réformes, les tenants du

\footnotetext{
${ }^{30}$ Plutôt « guerre contre les civils » (Tahon 1998).

${ }^{31}$ Les rapports des principales ONG de défense des droits de l'homme (Amnesty International, Fédération internationale des Droits de l'Homme, Human Rights Watch), qui ont toutes dépêché des missions d'enquête dans le courant de l'année 2000, sont accablants.

${ }^{32}$ «Cette loi ne vise que les combattants impliqués 'dans des actions de terrorisme ou de subversion'. Les membres des forces de l'ordre et des milices 'patriotiques' soutenues par le pouvoir (..) bénéficient d'une impunité de fait » (Kettani, 2001).
} 
pouvoir, dont la base sociale n'avait jamais été aussi réduite, ont sacrifié le développement à l'autel de leur maintien au pouvoir, et préféré l'ajustement structurel à une réforme menée de manière autonome et en toute souveraineté mais qui aurait nécessité d'ouvrir l'accès au champ politique. Cela les a menés à solliciter l'appui des puissances occidentales ${ }^{33}$, au moment précis où, après la chute du mur de Berlin, les rapports entre nations étaient reconfigurés à l'échelle planétaire. Vulnérabilisée, l'Algérie fut soumises à de multiples pressions de la part des puissances occidentales, visant entre autres, à sécuriser leur approvisionnement en pétrole et en gaz, ainsi qu'à déployer, de la Méditerranée à l'Afrique subsaharienne, un vaste dispositif de contrôle et d'endiguement des flux migratoires (Collyer, 2012). Si elle n'a pas été qu'un pion dans ces stratégies globalisées, la faction au pouvoir s'est surtout préoccupée d'user de sa marge de manœuvre pour obtenir en échange une reconnaissance internationale et un renforcement de sa position face à la contestation interne (Cavatorta, 2012), dans un conflit meurtrier qu'elle a cyniquement inscrit à l'agenda de la guerre mondiale contre le terrorisme (Keenan, 2012). Ce moment a été non seulement fatal au développement ${ }^{34}$, mais il a produit une fracture entre l'État et la société qui n'est pas refermée à ce jour.

Sur le plan institutionnel, rien n'a pu être réalisé depuis que la transition de la légitimité révolutionnaire issue de la guerre de libération (désormais épuisée) à une nouvelle légitimité sortie des urnes a été interrompue, laissant à l'abandon un chantier dévasté. Toute tentative de reconstruire sur ces ruines est vaine, car on ne peut édifier des institutions sans un socle de légitimité. C'est pour cette raison qu'aucun des grands chantiers législatifs ouverts n'a abouti, que ce soit la réforme des structures et missions de l'État ${ }^{35}$, la promulgation d'un nouveau code du travail annoncé comme imminente depuis 2006, la nouvelle stratégie industrielle élaborée en 2007 par le ministère de l'industrie et jamais adoptée officiellement, la loi sur les hydrocarbures de 2005, amendée par décret présidentiel moins d'un an après son adoption par l'assemblée nationale (Aïnas et al., 2012), les décrets présidentiels visant à mettre en conformité le droit interne avec les conventions internationales relatives aux droits des femmes ratifiées par l'Algérie, jamais mis en application; l'adhésion à l'OMC, en

\footnotetext{
33 « Entre la fin de 1993 et le début 1995, le régime algérien a pu bénéficier d'au moins 15 milliards de dollars à travers le rééchelonnement de la dette et des crédits de la part de gouvernements occidentaux, banques et organisations financières internationales » (Volpi, 2003).

${ }^{34}$ La part des hydrocarbures dans le PIB, qui était de $20 \%$ en 1988, dépasse $50 \%$ en 2006 . Alors qu'en 1988 son économie était encore relativement diversifiée, l'Algérie fait partie aujourd'hui des 10 pays à l'indice d'Herfindahl le plus élevé. Ses exportations sont parmi les moins diversifiés au monde, même au sein des pays pétroliers (Aïnas et al, 2012).

${ }^{35}$ La Commission de réforme des structures et missions de l'État, dite commission Sbih du nom de son président, créée fin 2000, a déposé un rapport en juillet 2001, non rendu public à ce jour.
} 
négociations depuis 1987 (GATT). A cela s'ajoute l'épineux dossier du foncier tant industriel qu'urbain et agricole, qui soulève la question des droits de propriété, sur laquelle on ne peut légiférer sans instance légitime. Malgré le recours à de coûteuses expertises, toutes ces réformes butent sur l'absence de légitimité des institutions, et en premier lieu de l'instance législative. Car la réforme des institutions n'est pas une affaire technique, ou du moins la technicité n'est pas suffisante.

Aujourd'hui, plus de douze ans après, les choses n'ont pas fondamentalement changé, l'impunité n'a pas été entamée, ce que seul permet d'expliquer le soutien dont jouit le régime à l'échelle internationale, en premier lieu de la part des puissances occidentales.

\section{Le printemps arabe en embuscade}

Le printemps arabe a remis à l'ordre du jour la question de l'impunité ainsi que celle du fondement démocratique des régimes, par le recours à la légitimité populaire sortie des urnes. Beaucoup se sont demandé pourquoi ne s'était-il pas étendu à l'Algérie ?

Plusieurs réponses ont été apportées à cette question : que l'Algérie a déjà accompli sa révolution démocratique en 1988, qu'elle sort à peine de longues années de violence... Si les années 1988-1991 ont effectivement représenté un espoir comparable à celui porté par le printemps arabe quant à une ouverture politique et de la société civile, les acquis de cette période ont été remis en cause et l'on a assisté à un immense bond en arrière. Le deuxième argument laisse supposer que l'Algérie a effectivement tourné la page de la violence, ce qui est malheureusement loin d'être le cas. Depuis que l'on a cessé de dénombrer les victimes de la guerre civile, rares sont les journées où la presse ne rapporte pas une ou plusieurs opérations - attentat ou action des forces de sécurité contre de présumés terroristes - avec leur

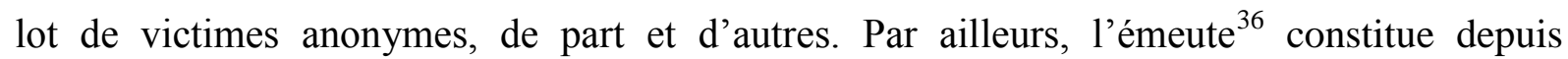
plusieurs années la forme privilégiée de la contestation sociale, et l'Algérie n'a rien à envier aux autres pays arabe en termes d'immolation par le feu, drame des harraga (Collyer, 2012) et autres actes de désespoir. A cela s'ajoute les formes de violence découlant de l'extension de zones de non-droit (criminalité et banditisme, réseaux de trafics divers..).

Pour répondre à cette question, il faudrait d'abord s'entendre sur l'analyse de ces soulèvements qui ont débuté en Tunisie en décembre 2010 et se sont étendus à la région, avec dans chaque cas de figure une configuration particulière. On peut dire globalement que ces

\footnotetext{
${ }^{36}$ Selon le directeur général de la sûreté nationale, 10910 interventions ont été effectuées en 2011 par les unités relevant des services de police, notamment de la sécurité publique, pour des situations de troubles à l'ordre public : regroupements, attroupements, obstructions de routes, sit in et marches non autorisées (Djazaïr.com, 6 janvier 2012).
} 
révoltes visaient partout le départ d'un dictateur détenant, souvent avec sa famille, le monopole du pouvoir (Benali en Tunisie, Kadhafi en Libye, Abdallah Saleh au Yémen..). Mais la fuite ou la mort d'un dictateur n'est que le début du processus, l'important étant que le montage institutionnel sur lequel il débouchera garantisse que l'horreur du régime déchu ne puisse se reproduise. Ainsi tous les yeux sont rivés sur l'issue des processus initiés en Tunisie et en Égypte.

En Algérie, le pouvoir est bicéphale: si le président dispose d'une certaine marge de manœuvre que lui confère sa fonction, mais surtout son habileté et sa ruse politique, il a en grande part les mains liées. Le pouvoir de l'ombre, celui du DRS ${ }^{37}$ (Keenan, 2012), tire sa force de sa capacité à mener des actions en toute illégalité et impunité avec les moyens de l'armée et de l'État, mais il doit user de stratagèmes et manipuler des civils quand il s'agit d'agir au niveau de la façade politique et des institutions. Il est à la fois l'héritier du régime antérieur $^{38}$ et le produit de la suspension du processus de démocratisation en $1992^{39}$.

\section{Conclusion}

Ainsi la rente pétrolière, dont la redistribution se fait à travers les circuits mis en place par le «pacte patrimonial», est certes un facteur de blocage, dans la mesure où sa persistance assure en quelque sorte la pérennité de ce pacte. Mais elle n'est pas une cause première. Nous pouvons tenter un rapprochement audacieux entre, d'une part, la tendance de la rente des hydrocarbures à recouvrir tout le champ économique, avec son corollaire, l'hégémonie du concept de rente et d'État rentier dans la littérature en sciences sociales et humaines sur l'Algérie, et, d'autre part, l'extension de ce pouvoir de l'ombre, qui agit dans l'illégalité et l'impunité. Il ne s'agit pas seulement de dire que celui-ci se maintient en prélevant sur la rente des hydrocarbures générée à l'extérieur. Mais aussi que son effet dissolvant du droit et de la légalité pousse à la dissolution des institutions, à l'informalisation de l'activité économique et finalement à l'effacement de la réalité de l'économie, de la société et des institutions - au sens où pour exister, toute chose doit advenir à la légalité, exister non seulement dans la langue, mais dans le droit (Supiot, 2009) - pour ne laisser apparaître comme seule réalité que la rente et l'État rentier. Dès lors, la rente peut être vue comme le symptôme de ce déni d'existence.

\footnotetext{
${ }^{37}$ Direction du Renseignement et de la Sécurité.

38 «La légalité socialiste n'instaurait pas le rule of law, mais, dans sa meilleure période (après la disparition de Staline), le rule by law » (Berman, $2003: 19$ ). «Sauf à qualifier de juridique n'importe quel système de règles, le système soviétique ne constituait pas un régime de Droit » (Supiot, $2010: 17$ ).

39 «La dissolution progressive du principe de légalité obtenu par des mesures sous forme de lois débouche sur une action criminelle hors de la loi et de toute forme légale » (Martin Broszat, cité par Supiot, 2010 : 16).
} 
Ce constat nous indique que le chemin vers la sortie de la rente et de l'État rentier, c'est-à-dire vers la réhabilitation de l'économie et des institutions, passe par un acte fondant dans les urnes une nouvelle légitimité.

\section{Références bibliographiques}

Acemoglu D., S. Johnson et J. A. Robinson (2001), « The Colonial Origins of Comparative Development. An empirical Investigation », American Economic Review, vol. 91, n5, pp. 1369-1401.

Aïnas Y., N.Ouarem et S. Souam (2012), «Les hydrocarbures : atout ou frein pour le développement de l'Algérie ?», Revue Tiers Monde, n²10, pp. 69-88.

Anderson L. (1987), « The State in the Middle East and North Africa », Comparative Politics, vol.20, n 1 , pp. 1-18.

Auty R. M. Et A. H. Gelb (2001), «Political Economy of Resource-Abundant State », in Auty R.M. (ed.), Resource Abundance and Economic Development, New York, Oxford University Press.

Beblawi Hazem, (1990), « The Rentier State in the Arab World », in G. Luciani (ed.), The Arab State, Londres, Routledge, pp. 85-98.

Beblawi H. et G. Luciani (eds.) (1987), The Rentier State, Croom Helm.

Bellache Y. (2010), L'économie informelle en Algérie, une approche par enquête auprès des ménages. Le cas de Bejaia, thèse de doctorat en sciences économique, Université de Bejaia (Algérie)/Université Paris -Est Créteil (France).

Bevan D., P. Collier et J. W. Gunning (1990), Controlled Open Economy. A neo-classical Approach to structuralism, Oxford, Clarendon Press. 
Berman H. (2003), Law and Revolution, t.2, Havard University Press. Bhabwati J. (1982), «Directly Unproductive Profit-seeking (DUP) Activities », Journal of Political Economy, vol. $90, \mathrm{n}^{\circ} 5$, pp. 988-1002.

Bhagwati J. (1996), Political Economy and International Economy, USAA, MIT Press.

Bouklia-Hassane R. et F. Talahite (2008), « Marché du travail, régulation et croissance économique en Algérie », Revue Tiers Monde, t. XLIX, nº194.

Buchanan J. M. (1980), « Rent Seeking and Profit Seeking» in J. M. Buchanan, R. D. Tollison et G. Tullock (eds.), Toward a Theory of the Rent-Seeking Society, College Park, Texas, A\&M Press.

Catusse M. (2006), «Ordonner, classer, penser la société: les pays arabes au prisme de l'économie politique », in E. Picard (ed.), Le politique dans le monde arabe, Paris, A. Colin, pp. 215-238.

Cavatorta F. (2012), «La reconfiguration des structures de pouvoir en Algérie », Revue Tiers Monde, $\mathrm{n}^{\circ} 210$, pp. 13-29.

Collyer M. (2012), «Moving Targets : Algerian State Responses to the Challenge of

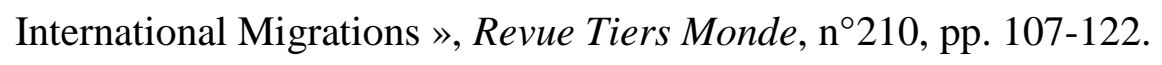

Dillman B. L. (2000), State and Private Sector in Algeria : The Politics of Rent-Seeking and Failed Development, San Francisco/Oxford, Boulder/Westview Press.

Hadj-Nacer A. (dir.) (1989, 1990), Les cahiers de la réforme, Alger, ENAG éditions, ${ }^{\circ} 1$ à 5.

Hadj-Nacer A. (2011), La martingale algérienne : réflexion sur une crise, Alger, éditions Barzakh.

Henni A. (1999), «Economie parallèle : une union rampante », Panoramique, n41. 
Henni A. (2009), «La réforme monétaire et financière en Algérie: enseignements pour une transition vers le marché dans un pays en voie de développement », Confluence Méditerranée, $\mathrm{n}^{\circ} 71$, pp. $27-40$.

Hicks J. R. (1969), A Theory of Economic History, Oxford University Press. Traduction française, Une théorie de l'histoire économique, Seuil 1973.

Keenan J. (2012), «Politique étrangère et guerre mondiale contre le terrorisme dans la reproduction du pouvoir algérien », Revue Tiers Monde, n²10, pp. 31-50.

Kettani K. (2001), «Algérie: concorde civile, impunité et droit international », Sanabil, Journal électronique pour un Maghreb des droits de l'Homme, $\mathrm{n}^{\circ} 1$, mars. http://www.maghreb-ddh.sgdg.org/sanabil/numero1

Khan M. H. et K. S. Jomo (2000), Rents, Rent-Seeking and Economic Development: Theory and Evidence in Asia, Cambridge University Press.

Kornaï J. (1990), The Road to a Free Economy from a Socialist System. The Example of Hungary, Traduction française (1990) Du socialisme au capitalisme. L'exemple de la Hongrie, Paris, Gallimard.

Krueger A. (1974), «The Political Economy of the Rent-Seeking Society », American Economic Review vol. 64, n³, pp. 291-303

Legendre P. (1999), Sur la question dogmatique en Occident, Paris, Fayard.

Mahdavi H. (1970), «The Patterns and Problems of Economic Development in Rentier States : the Case of Iran », in M.A. Cook (ed.), Studies in the Economic History of the Middle East, Londres, Oxford University Press, pp. 428-467.

Marx K. (1965), Euvres, Économie, tome 1, Paris, La Pléïade.

Omari C., J.-Y. Moisseron, A. Alpha (2012), «La reconfiguration des structures de pouvoir en Algérie », Revue Tiers Monde, n²10, pp. 123-141. 
Rivlin P. (2009), Arab Economies in the Twenty-First Century, Cambridge, Cambridge University Press.

Robertson R.G. (2005), Conquest by Law. How the Discovery of America Dispossessed Indigeanous Peoples of Their Lands, Oxford/NewYork, Oxford University Press.

Safar Zitoun M. (2012), «Etat providence et politique du logement en Algérie », Revue Tiers Monde, n²10, pp. 89-106.

Sgard J. (1997), Europe de l'Est, la transition économique, Paris, Flammarion.

Supiot A. (2009), Homo juridicus : Essai sur la fonction anthropologique du Droit, Paris, Points.

Supiot A. (2010), L'esprit de Philadelphie : La justice sociale face au marché total, Paris, Seuil.

Talahite F. (2006), « Le concept de rente : pertinence et dérives », Problèmes économiques, $\mathrm{n}^{\circ} 2.908$.

Talahite F. et R. Bouklia-Hassane (2012), «Les politiques industrielles en Algérie (19632007) », in 0. Orozco (ed.), Politiques industrielles au Maghreb, Madrid, Casa Arabe.

Talahite F. et A. Hammadache (2011), «L'économie algérienne d'une crise à l'autre », in Maghreb-Machrek, n²06, pp. 101-123.

Tahon M.-B. (1998), Algérie, la guerre contre les civils, Québec, Nota Bene.

Tullock G. et G. Mason (2005), Public goods, redistribution and rent seeking, Cheltenham (UK)/Northampton (MA), The Locke Institute/Edward Elgar.

Volpi F. (2003), Islam and Democracy :the Failure of Dialogue in Algeria, Londres, Pluto Press. 
Weber A.-P. (2010), 1830-1930, La France en Algérie: une mauvaise aventure, Paris, Publibook.

Williamson J. G. (2011), Trade and Poverty. When the Third World Fell Behind, Cambridge, The MIT Press.

Woolcock M., L. Pritchett et J. Isham (2001), « The Social Foundations of Poor Economic Growth », in R.M. Auty (ed.), Resource Abundance and Economic Development, Oxford University Press, pp. 76-92. 